\title{
Efficacy of the Thoracolumbar Interfascial Plane Block for Lumbar Laminoplasty: A Retrospective Study
}

\author{
Hironobu Ueshima ${ }^{1}$, Tomoyuki Ozawa ${ }^{2}$, Tomoaki Toyone ${ }^{2}$, Hiroshi Otake ${ }^{1}$ \\ ${ }^{1}$ Department of Anesthesiology, Showa University Hospital, Tokyo, Japan \\ ${ }^{2}$ Department of Orthopedics, Showa University Hospital, Tokyo, Japan
}

Study Design: This paper was a single center-based retrospective study with prospective data collection.

Purpose: Compared with other surgeries, limited options are available for perioperative pain management in spinal surgery. Therefore, we aimed to identify new pain management in this study.

Overview of Literature: The thoracolumbar interfascial plane (TLIP) block has been reported to provide effective regional analgesia in the lumbar region. This study investigated the efficacy of the TLIP block for pain management in lumbar laminoplasty.

Methods: We investigated patients who underwent lumbar laminoplasty for the treatment of lumbar spinal canal stenosis from April to October 2015. Patients with secondary surgery or surgery involving more than four intervertebral spaces were excluded. The primary outcome measure was the pain scale score within 48 hours after the surgery. The secondary outcomes were the number of additional analgesic drugs used and the number of patients complaining of complications, such as nausea and vomiting, within 24 hours after the surgery.

Results: We retrospectively assessed the data of 44 patients who underwent lumbar laminoplasty. Of these, 25 patients received only general anesthesia ( $G$ group), whereas 19 patients received the TLIP block along with general anesthesia (T group). Compared with the $\mathrm{G}$ group, the T group reported lower pain scores for pain at 1, 2, 4, and 24 hours postoperatively. Moreover, the number of patients who received the additional analgesic pentazocine was lower in the T group than in the $\mathrm{G}$ group. The two groups showed no significant differences in the incidence of complications.

Conclusions: The TLIP block provides effective analgesia for 24 hours postoperatively in patients undergoing lumbar laminoplasty.

Keywords: Nerve block; Lumbar; Pain management

\section{Introduction}

Although the number of spinal surgeries performed has long increased $[1,2]$, the methods available for perioperative pain management have remained limited. The thoracolumbar interfascial plane (TLIP) block is performed by injecting a local anesthetic drug into the fascial plane between the multifidus and longissimus muscles at the approximate level of the third lumbar vertebra. It has been reported to block the dorsal rami of the thoracolumbar nerves as they pass through the paraspinal musculature $[3,4]$. However, no clinical studies have reported the ef-

Received Feb 1, 2017; Revised Feb 23, 2017; Accepted Mar 19, 2017

Corresponding author: Hironobu Ueshima

Department of Anesthesiology, Showa University Hospital, 1-5-8 Hatanodai Shinagawa-ku, Tokyo 142-8666, Japan

Tel: +81-03-3784-8875, Fax: +81-03-3784-8357, E-mail: ueshimhi@yahoo.co.jp 
ficacy of the TLIP block for lumbar surgery [4]. In this study, we retrospectively investigated the efficacy of the TLIP block for lumbar laminoplasty.

\section{Materials and Methods}

This retrospective study was approved by the Showa University Hospital Institutional Review Board (approval no., 1953). In addition, the study was registered at the University Hospital Medical Information Network (UMIN ID no., 000020015). We included patients who underwent lumbar laminoplasty for the treatment of lumbar spinal canal stenosis from April to October 2015. Cases of secondary surgery and surgeries involving more than four intervertebral spaces were excluded. Preoperative data, including the data of age, height, weight, American Society of Anesthesiologist classification, surgery time, anesthesia time, and amount of fentanyl and remifentanil administered in the perioperative period, were collected. All patients were moved to the high-care unit at our hospital within 48 hours after the surgery. The primary outcome measure was the pain scale score within 48 hours after the surgery. Each patient's pain level was measured at rest by two nurses at 1, 2, 4, 6, 12, 24, and 48 hours postoperatively. The postoperative pain score was assessed using a visual analog scale $(0-100 \mathrm{~mm}$, where $0=$ no pain and $100=$ most severe pain). Patients received $15 \mathrm{mg}$ of intravenous pentazocine for complaints of pain, regardless of the pain level. In addition, patients were administered with acetaminophen 1,000 mg intravenously three times a day. If both were unable to control the pain, an attending anesthesiologist was consulted. After consultation, the patient was prescribed a continuous intravenous administration of fentanyl.

Patients were injected with $10 \mathrm{mg}$ of intravenous metoclopramide for complaints of nausea and vomiting.

\section{TLIP block technique}

The TLIP block was performed by injecting $40 \mathrm{~mL}$ of $0.375 \%$ levobupivacaine ( $20 \mathrm{~mL}$ on each side) into the fascial plane between the multifidus and longissimus muscles by using a high-frequency linear probe. The anesthetic was injected at the approximate level of the third lumbar vertebra (Fig. 1).

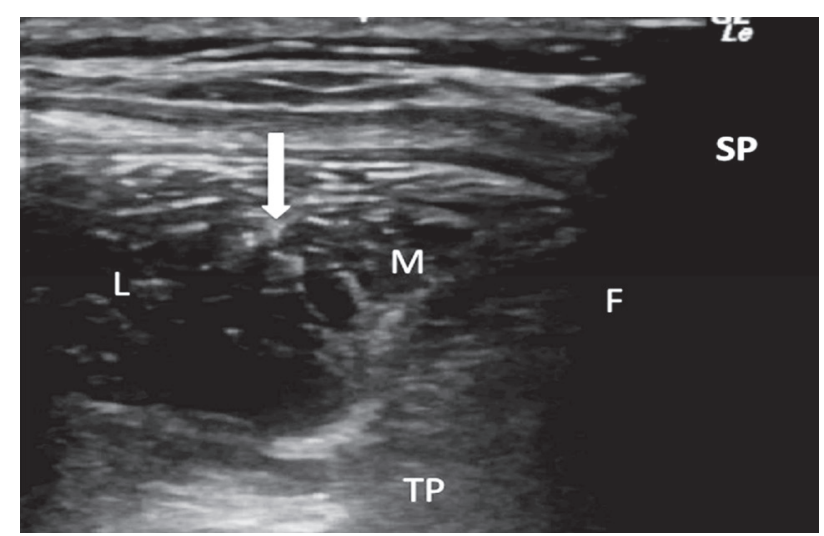

Fig. 1. Ultrasound image of the paraspinal muscles of the lumbar spine. The point of thoracolumbar interfascial plane block is indicated by a white arrow in the ultrasound image. SP, spinous process in L3; F, facet joint; $L$, longissimus muscle; $M$, multifidus muscle; TP, transverse process.

\section{Statistical analysis}

Statistical analysis was performed using JMP 12 (SAS Institute Inc., Cary, NC, USA). The data of pain scores and number of pentazocine injections in one patient were analyzed using the Mann-Whitney $U$ test. The metoclopramide injection data were analyzed using the Fisher exact test. Data are expressed as median (range). The level of significance for both tests was set at $p<0.05$.

\section{Results}

In this study, 45 patients who underwent lumbar laminoplasty for the treatment of lumbar spinal canal stenosis were evaluated. One patient was excluded because of secondary surgery. Finally, the remaining 44 patients were divided into two groups: 25 patients received only general anesthesia ( $G$ group), whereas the other 19 patients received a TLIP block along with general anesthesia (T group). Table 1 shows the comparison of the patients' demographic data, and Table 2 shows the contents of surgical procedures according to the groups. Compared with the $\mathrm{G}$ group, the $\mathrm{T}$ group exhibited lower pain scores at 1 , 2,4 , and 24 hours postoperatively (Table 3 ). The number (1 [0-2]) of pentazocine injections administered in the T group was lower than that $(1[1-3])$ administered in the $\mathrm{G}$ group ( $p=0.04)$. No patients were prescribed a continuous intravenous administration of fentanyl. Four patients in the $\mathrm{G}$ group and three patients in the T group experienced complications. However, the two groups showed no significant differences in the incidence of complications such 
Table 1. Patients demogra phics data

\begin{tabular}{lccc} 
Variable & G group $(\mathrm{n}=25)$ & T group (n=19) & $p$-value \\
\hline Age $(\mathrm{yr})$ & $71(26-83)$ & $68(48-83)$ & 0.65 \\
\hline Height $(\mathrm{cm})$ & $159(149-177)$ & $166(146-175)$ & 0.74 \\
\hline Weight $(\mathrm{kg})$ & $61(39-83)$ & $65(40-108)$ & 0.81 \\
\hline ASA classification (I/II/III) & $3 / 20 / 2$ & $5 / 11 / 3$ & - \\
\hline Surgery time (min) & $185(80-335)$ & $180(65-300)$ & 0.98 \\
\hline Anesthesia time $(\mathrm{min})$ & $225(150-355)$ & $220(120-385)$ & 0.98 \\
\hline Fentanyl $(\mu \mathrm{g})$ & $200(100-300)$ & $200(100-400)$ & 0.30 \\
\hline Remifentanil $(\mu \mathrm{g})$ & $0.25(0.1-1.0)$ & $0.2(0.1-0.6)$ & 0.15 \\
\hline Values & & & \\
\hline
\end{tabular}

Values are presented as a median (range).

G, only general anesthesia; T, thoracolumbar interfascial plane block with general anesthesia; ASA, American Society of Anesthesiologists.

Table 2. The contents of surgical procedure

\begin{tabular}{lcc} 
At the site of lumbar laminoplasty & $\mathrm{G}$ group $(\mathrm{n}=25)$ & T group (n=19) \\
L1/2 & 0 & 0 \\
\hline L2/3 & 0 & 0 \\
$L 3 / 4$ & 7 & 6 \\
$L 4 / 5$ & 7 & 5 \\
$L 5 / S 1$ & 2 & 0 \\
$L 1 / 2 / 3$ & 0 & 0 \\
$L 2 / 3 / 4$ & 7 & 1 \\
$L 3 / 4 / 5$ & 2 & 6 \\
$L 4 / 5 / S 1$ & 0 & 1 \\
\hline
\end{tabular}

G, only general anesthesia; T, thoracolumbar interfascial plane block with general anesthesia.

Table 3. Postoperative pain scores at 1, 2, 4, 6, 12, 24, and 48 postoperative hours during the rest

\begin{tabular}{|c|c|c|c|}
\hline Postoperative hours (hr) & T group $(n=25)$ & $\mathrm{G}$ group $(\mathrm{n}=19)$ & $p$-value \\
\hline 1 & $30(15-45)$ & $35(20-80)$ & 0.03 \\
\hline 2 & $35(25-55)$ & $40(25-60)$ & 0.04 \\
\hline 4 & $40(25-55)$ & $45(25-60)$ & 0.04 \\
\hline 6 & $35(30-45)$ & $45(30-60)$ & 0.05 \\
\hline 12 & $30(25-50)$ & $40(25-60)$ & 0.08 \\
\hline 24 & $30(20-45)$ & $40(25-55)$ & 0.01 \\
\hline 48 & $40(25-45)$ & $40(25-55)$ & 0.45 \\
\hline
\end{tabular}

Values are presented as a median (range).

T, thoracolumbar interfascial plane block with general anesthesia; $G$, only general anesthesia.

as nausea and vomiting $(p=0.65)$.

\section{Discussion}

We demonstrated that the TLIP block affects the dorsal rami of the lumbar nerves and can provide effective anal- gesia for 24 hours after lumber laminoplasty [3,4]. In our cadaveric study, the dye added to the anesthetic of the TLIP block colored the dorsal rami of the lumbar nerves [5].

Hand et al. [3] reported that the efficacy of the TLIP block is restricted to the lumbar region; however, we determined that the TLIP block also affected the dorsal 
rami of the thoracic nerves [6]. By identifying the fascial plane between the multifidus and longissimus muscles, we can block the wide dorsal rami from the cervical to lumbar nerves. Furthermore, the TLIP block may be safer and easier to perform than epidural anesthesia, the paravertebral nerve block, and the retrolaminar block. This is because the TLIP block is administered more superficially [7-10]. Therefore, it may become the preferred nerve block for spinal surgery in the future.

There are some limitations of this study. Because this was a retrospective study, the data were not randomized. In addition, we could not measure the anatomic location of the drug effect. Furthermore, we do not know the ideal volume and concentration of anesthetic required to perform the TLIP block. Currently, we have used an amount of local anesthetic that would not cause systemic toxicity. Moreover, this study focused on outcomes from only the first 48 hours after surgery, and the efficacy of the TLIP block for chronic pain remains unknown. Therefore, a prospective study should be conducted in the near future to overcome these limitations.

Finally, effective analgesia in the perioperative period may prevent the development of chronic pain, and the TLIP block can play a role in achieving this result [11].

\section{Conclusions}

The TLIP block provides effective analgesia for the first 24 hours postoperatively in patients undergoing lumbar laminoplasty.

\section{Conflict of Interest}

No potential conflict of interest relevant to this article was reported.

\section{References}

1. Weinstein JN, Lurie JD, Olson PR, Bronner KK, Fisher ES. United States' trends and regional variations in lumbar spine surgery: 1992-2003. Spine (Phila Pa 1976) 2006;31:2707-14.

2. Rajaee SS, Bae HW, Kanim LE, Delamarter RB. Spinal fusion in the United States: analysis of trends from 1998 to 2008. Spine (Phila Pa 1976) 2012;37:6776.

3. Hand WR, Taylor JM, Harvey NR, et al. Thoracolumbar interfascial plane (TLIP) block: a pilot study in volunteers. Can J Anaesth 2015;62:1196-200.

4. Ueshima H, Sakai R, Otake H. Clinical experiences of ultrasound-guided thoracolumbar interfascial plane block: a clinical experience. J Clin Anesth 2016;33:499.

5. Ueshima H, Oku K, Otake H. Ultrasound-guided thoracolumbar interfascial plane block: a cadaveric study of the spread of injectate. J Clin Anesth 2016;34:259-60.

6. Ueshima H, Otake H. Clinical efficacy of modified thoracolumbar interfascial plane block. J Clin Anesth 2016;30:74-5.

7. Ulutas M, Secer M, Taskapilioglu O, et al. General versus epidural anesthesia for lumbar microdiscectomy. J Clin Neurosci 2015;22:1309-13.

8. Karmakar MK, Gin T, Ho AM. Ipsilateral thoracolumbar anaesthesia and paravertebral spread after low thoracic paravertebral injection. Br J Anaesth 2001;87:312-6.

9. Voscopoulos C, Palaniappan D, Zeballos J, Ko H, Janfaza D, Vlassakov K. The ultrasound-guided retrolaminar block. Can J Anaesth 2013;60:888-95.

10. Murouchi T, Yamakage M. Retrolaminar block: analgesic efficacy and safety evaluation. J Anesth 2016;30:1003-7.

11. Kairaluoma PM, Bachmann MS, Rosenberg PH, Pere PJ. Preincisional paravertebral block reduces the prevalence of chronic pain after breast surgery. Anesth Analg 2006;103:703-8. 